\title{
EGU2020-22390
}

https://doi.org/10.5194/egusphere-egu2020-22390

EGU General Assembly 2020

(c) Author(s) 2020. This work is distributed under

the Creative Commons Attribution 4.0 License.

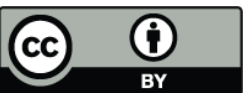

\section{Mars Express Science Highlights and Future Plans}

Dmitrij Titov, Jean-Pierre Bibring ${ }^{2}$, Alejandro Cardesin ${ }^{3}$, Thomas Duxbury ${ }^{4}$, Francois Forget ${ }^{5}$, Marco Giuranna ${ }^{6}$, Francisco Gonzailez-Galindo ${ }^{7}$, Mats Holmström ${ }^{8}$, Ralf Jaumann ${ }^{9}$, Anni Määttänen ${ }^{10}$, Patrick Martin ${ }^{3}$, Franck Montmessin ${ }^{10}$, Roberto Orosei ${ }^{11}$, Martin Pätzold ${ }^{12}$, and Jeff Plaut ${ }^{13}$

${ }^{2}$ IAS-CNRS, Orsay, France

${ }^{3}$ ESA-ESAC, Madrid, Spain

${ }^{4}$ George Mason University, Fairfax, VA, USA

${ }^{5}$ LMD, Paris, France

${ }^{6}$ IAPS-INAF, Rome, Italy

${ }^{7}$ IAA, Granada, Spain

${ }^{8} \mathrm{IRF}$, Kiruna, Sweden

${ }^{9}$ IPF-DLR, Berlin, Germany

${ }^{10}$ LATMOS/ IPSL, CNRS, Guyancourt, France

${ }^{11}$ IRA-INAF, Bologna, Italy

${ }^{12}$ RIU-Uni Cologne, Cologne, Germany

${ }^{13} \mathrm{JPL}$, Pasadena, CA, USA

After 16 years in orbit Mars Express remains one of ESA's most scientifically productive Solar System missions which publication record now exceeds 1270 papers. Characterization of the geological processes on a local-to-regional scale by HRSC, OMEGA and partner experiments on NASA spacecraft has allowed constraining land-forming processes in space and time. Recent studies suggest geological evidence of a planet-wide groundwater system on Mars and surface clay formation during short-term warmer and wetter conditions on a largely cold ancient Mars that might indicate a change in our understanding of early Mars climate. HRSC team released first set of multi-orbit Digital Elevation Model (DEM) of the MC-11 quadrangle and the Southern polar cap with $50 \mathrm{~m} / \mathrm{px}$ resolution. Mars Express observations and experimental teams provided essential contribution to the selection of the Mars-2020 landing sites and supporting characterization of potential landing sites for the Chinese HX-1 mission. Following recent discovery of subglacial liquid water underneath the Southern polar layered deposits the MARSIS radar continues searching for subsurface water pockets.

One-and-half decade of monitoring of atmospheric parameters such as temperature, dust loading, water vapor and ozone abundance, water ice and $\mathrm{CO} 2$ clouds distribution, collected by SPICAM, PFS, OMEGA, HRSC and VMC together with subsequent modeling have provided key contributions to our understanding of the Martian climate. The observed ozone climatology demonstrate significant discrepancies with model predictions indicating the need for models improvement. In 2018 PFS confirmed observations of a methane abundance "spike" in the Gale crater observed in 
situ by the Curiosity Rover. Recent similar quasi-simultaneous observations were in disagreement, thus indicating that the methane "enigma" continues. This poses a significant challenge to both observers and modelers. The radio-science experiment MaRS revealed fine structure of the boundary layer. Its depth varies from $2 \mathrm{~km}$ in topographic lows to $\sim 10 \mathrm{~km}$ over highlands.

Observations of the ion escape during complete solar cycle revealed that ion escape can be responsible for removal of about 10 mbar over Mars history that implies existence of other more effective escape channels.

The structure of the ionosphere derived from MARSIS and MaRS sounding was found to be significantly affected by the solar activity, the crustal magnetic field. The observations suggest that the sunlit ionosphere over the regions with strong crustal fields is denser and extends to higher altitudes as compared to the regions with no crustal anomalies. Expansion of the ionosphere was also observed during the global dust storm. lonospheric models aim at creating user-friendly data base of plasma parameters that would be of great service to the planetary community.

The "gyroless" attitude control and operations mode of the spacecraft operates flawlessly since April 2018. Aging batteries impose more and more limitations on science operations during eclipse seasons. The mission is now confirmed till the end of 2020 and notionally extended till the end of 2022. The talk will give the Mars Express status, review the recent science highlights, and outline future plans including synergistic science with TGO. 